\title{
Primer estudio sistemático de la colonia reproductiva de albatros de ceja negra Thalassarche melanophris (Temminck, 1828) en el Seno Almirantazgo, Tierra del Fuego (Chile)
}

\author{
DANIELA DROGUETT ${ }^{1} \bowtie$, ALEJANDRO VILA², ALEJANDRO KUSCH ${ }^{3}$, \\ RICARDO MATUS ${ }^{4}$, BENJAMÍN CÁCERES 5 Y JAVIER ARATA 6 \\ 1.https://orcid.org/0000-0002-2627-3790, ddroguett@wcs.org; \\ 2. https://orcid.org/0000-0001-5482-3852; 3. https://orcid.org/0000-0001-7476-5776; \\ 4. https://orcid.org/0000-0001-6636-3523; 5. https://orcid.org/0000-00026356-3770; 6. https://orcid.org/0000-0001-7320-0511
}

\section{OPEN ACCESS}

\section{Recibido:}

21/08/2021

Revisado:

18/10/2021

\section{Aceptado:}

05/11/2021

Publicado en línea:

31/12/2021

\section{Editor asociado:}

Dr. Américo Montiel San Martín.

ISSN 0718-686X
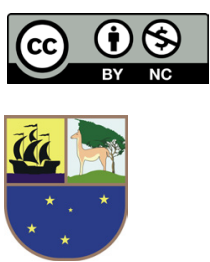

\section{RESUMEN}

Las colonias reproductivas de albatros de ceja negra (Thalassarche melanophris) se reconocen por su gran tamaño y presencia en islas oceánicas. Sin embargo, en el año 2003 se describe por primera vez la existencia de la única, y numéricamente pequeña, colonia reproductiva en aguas interiores de la Patagonia chilena ubicada en el islote Albatros ( $54^{\circ} 27^{\prime} 20^{\prime \prime}$ S; $69^{\circ} 01^{\prime} 12^{\prime \prime} \mathrm{W}$ ). A partir del año 2009 , se inicia un estudio sistemático de esta colonia, visitando el área durante la temporada reproductiva para monitorear y evaluar su población. Se realizaron once expediciones entre los meses de octubre y febrero, durante los años 2009 y 2016, contabilizando la presencia de huevos y polluelos para determinar el éxito reproductivo de la colonia. Adicionalmente se anilló un total de 188 individuos a lo largo de 7 temporadas para determinar la tasa de recaptura durante estos años. A partir de estos monitoreos, se espera aportar nuevos antecedentes al conocimiento general sobre la pequeña colonia de albatros de ceja negra, única en el mundo ubicada en aguas interiores de la Región de Magallanes y de la Antártica Chilena.

Palabras clave: Reproducción, aguas interiores, Tierra del Fuego, Área Marina Protegida. 
CÓMO CITAR:

Droguett et al

Primer estudio sistemático de la colonia reproductiva de albatros de ceja negra Thalassarche melanophris (Temminck, 1828)

en el Seno Almirantazgo Tierra del Fuego (Chile) 49. https://doi.org/10.22352/ AIP202149014

\section{Contribución} de los autores:

DD: Redacción y edición del manuscrito, análisis de la información

elaboración de figuras confeccion de tablas,toma de datos en terreno.

AV.: Diseño de la metodologia en terreno, toma de datos en terreno, edición del manuscrito analisis de datos.

AK.: Revisión de texto del MS. Trabajo en terreno datos en terreno.

RM.: Trabajo en terreno, metodologia de anillamiento, Revision del texto del MS, anillamiento.

BC: Trabajo en terreno,Revisión del texto del MS

JA.: Contribuciones al diseño de la metologia en terreno, analisis de datos, confeccion de tablas y figuras edicion y revision del MS.

Declaración de interéses:

Los autores declaran no tener conflicto de intereses.

Financiamiento: Ministerio del Medio Ambiente, Wildlife Conservation Society Chile y The David and Lucile Packard Fundation

\section{First systematic study of the breeding colony of black-browed albatrosses Thalassarche melanophris (Temminck, 1828) in admiralty sound, Tierra del Fuego (Chile)}

\section{ABSTRACT}

Breeding colonies of black-browed albatrosses (Thalassarche melanophris) are recognized by their large size and existence on oceanic islands. However, in 2003 the existence of the only numerically small reproductive colony in inland waters within Chilean Patagonia was described for the first time in the Albatross Islet ( $54^{\circ} 27^{\prime} 20^{\prime \prime} \mathrm{S} ; 69^{\circ} 01^{\prime} 12^{\prime \prime} \mathrm{W}$ ). Starting in 2009 , we began a systematic study of this colony, visiting the area during the reproductive season to monitor and evaluate its population. Eleven expeditions were carried out between the months of October and February, amid the years 2009 and 2016, counting the presence of eggs and chicks to determine the reproductive success of the colony. Additionally, a total of 188 individuals were banded over 7 seasons to determine their recapture rate during these years. With these monitoring, it is expected to contribute new information to the general knowledge about the small colony of black-browed albatrosses, the only one in the world located in inland waters in the Magallanes Region and the Chilean Antarctic.

Key words: Breeding, inland waters, Tierra del Fuego, Marine Protected area 


\section{INTRODUCCIÓN}

El albatros de ceja negra (Thalassarche melanophris) presenta una distribución circumpolar, se reproduce anualmente, posee una alta tasa de sobrevivencia de adultos, un bajo éxito reproductivo anual y una maduración sexual tardía (Nevoux et al. 2007; Weimerskirch et al. 1987), ingresando en promedio a la vida reproductiva a los 10 años de edad (Prince et al. 1994). Su período reproductivo ocurre entre los meses de octubre a abril de cada año (Croxall et al. 1998; Prince et al. 1994). La incubación dura 68 días, durante la cual ambos padres se turnan, realizando relevos de hasta dos semanas (Campioni et al. 2017). Los padres realizan largos viajes en búsqueda de alimento, particularmente durante la incubación. Por el contrario, como en otras aves marinas, la duración de estos viajes y la distancia recorrida durante la crianza están muy restringidas (Arata et al. 2014; Wakefield et al. 2011).

Esta especie nidifica, principalmente, en islas subantárticas expuestas del Atlántico suroccidental y el Pacífico suroriental (Robertson et al. 2007), las cuales concentran el $86 \%$ de su población global (Arata et al. 2009). Algunas de las colonias son de gran tamaño, como las de las islas Falkland/Malvinas, con aproximadamente 475.500 a 535.000 parejas (Wolfaardt, 2012).

En Chile nidifica el 21\% de la población mundial (Arata et al. 2009; Foro para la Conservación del Mar Patagónico y áreas de influencia, 2019) distribuida en seis áreas de nidificación, con un total de parejas reproductivas estimado en 122.920 (Moreno \& Robertson, 2008; Ojeda et al. 2011). Las principales áreas de nidificación en Chile corresponden a las islas oceánicas subantárticas como Diego Ramírez, Diego de Almagro e lldefonso, las cuales cumplen un rol relevante para la conservación de aves marinas a nivel global (Robertson et al. 2017).

Durante los años 2003 y 2007 se identificaron 3 colonias nuevas para la especie en los islotes Evangelistas (4.670 parejas reproductivas; Arata et al. 2003), islote Leonard (594 parejas reproductivas; Marín \& Oehler, 2007) y la única colonia de aguas interiores del mundo, el islote Albatros, con 50 parejas y 40 nidos ocupados por adultos en el mes de enero (Aguayo et al. 2003). Moreno y Robertson (2008) y Robertson y colaboradores (2014), basados en censos aéreos, determinaron la presencia de 65 y 111 parejas, en octubre de 2006 y 2011, respectivamente, en este islote.

En términos de conservación de esta especie en Chile, se considera como una especie de Preocupación Menor, siendo la mortalidad incidental en operaciones de pesca comercial, una de las amenazas más comunes que enfrenta (Robertson et al. 2014). Sin embargo, la implementación de medidas de mitigación para disminuir los efectos de la pesca incidental ha tenido efectos positivos en las principales colonias (Moreno \& Robertson, 2008; Robertson et al. 2014). A diferencia de lo que ocurre en las otras colonias de la especie, los individuos del islote Albatros se alimentan principalmente en los fiordos y canales interiores de la región de Magallanes (Arata et al. 2014), donde no opera la flota industrial de pesca.

Esta investigación tiene como objetivo evaluar y determinar el tamaño de la colonia, su fenología y su éxito reproductivo. Estos registros aportarán a futuras investigaciones y decisiones de manejo sobre esta especie, considerando que tanto el área de alimentación como de reproducción del albatros de ceja negra han sido identificados como elementos prioritarios de conservación del Área Marina Costera Protegida de Múltiples Usos (AMCP-MU) Seno Almirantazgo (Vila et al. 2017) en Tierra del Fuego, Chile. 
Fig. 1.

a) Región de Magallanes y de la Antártica Chilena. Área Marina Costera Protegida de Múltiples Usos Seno Almirantazgo

(AMCP-MU, área oscura en el mapa).

b) Ubicación del islote Albatros en el AMCP-MU en Tierra de Fuego, Chile.

c) Islote Albatros. La flecha indica la ubicación de la colonia (Créditos fotografía: Luis Bertea).
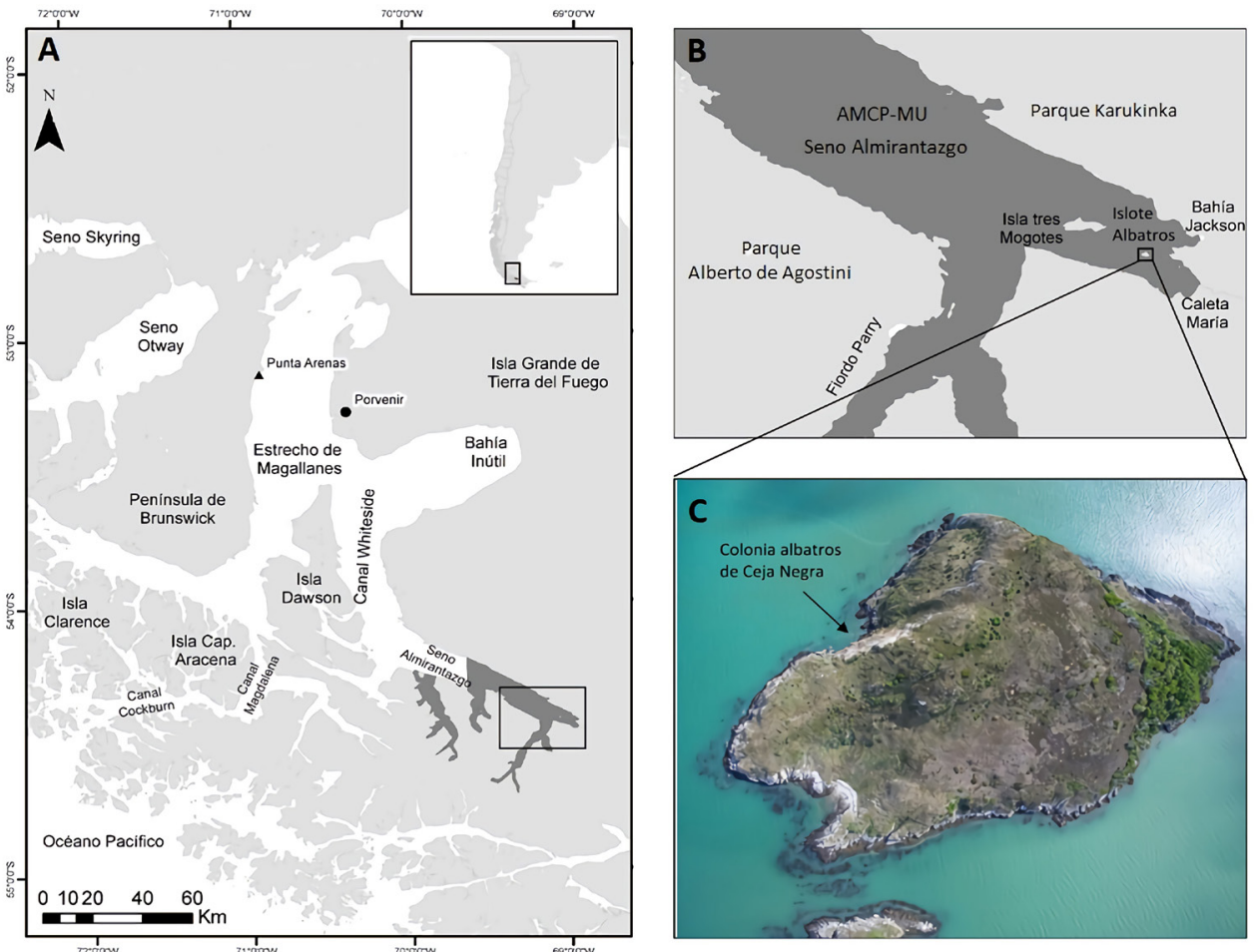

\section{MÉTODOS}

\section{Área de estudio}

El islote Albatros se ubica en Tierra del Fuego, Chile, dentro del AMCP-MU Seno Almirantazgo $\left(54^{\circ} 27^{\prime} 20^{\prime \prime} \mathrm{S} ; 69^{\circ} 01^{\prime} 12^{\prime \prime} \mathrm{W}\right.$ ) y tiene una superficie de $11,83 \mathrm{ha}$. La colonia de albatros de ceja negra se ubica en la ladera norte (Fig. 1), está expuesta a los vientos predominantes del NW y sobre una pendiente de unos $30^{\circ}$ a $40^{\circ}$ (Aguayo et al. 2003).

\section{Registro de datos}

Se colectaron datos durante siete temporadas, entre los años 2009 y 2016 (Tabla 1). Considerando la temporalidad reproductiva de la especie, las expediciones se realizaron entre los meses de octubre y febrero (Fig. 2). En cada temporada se realizó un conteo directo del número de "nidos vacíos" (nidos armados, pero sin presencia de adultos, huevos o pollos) y de "nidos activos", nidos con presencia de huevo o polluelos (Fig. 3a). Adicionalmente, se marcaron los nidos activos con chapas numeradas (Fig. 3b) para su monitoreo a largo plazo. A partir de estos datos se estimó la fecha media de eclosión. Para ello, se consideró el día 14 de diciembre como la fecha de eclosión, teniendo en cuenta la duración del período de incubación (Campioni et al. 2017), se estimó la fecha de postura 68 días antes de la eclosión (7 de octubre). Se estimó la tasa de sobrevivencia de huevos (período de incubación) y polluelos (desde eclosión en adelante) utilizando los datos combinados de todas las temporadas.

Se utilizó Kruskal-Wallis como test estadístico. 
Fig. 2.

Ciclo reproductivo del albatros de ceja negra, basado en imágenes del islote Albatros, seno Almirantazgo

(Créditos fotográficos: Archivo WCS Chile)

Fig. 3.

a) Registro de datos en los nidos.

b) Adulto nidificando en nido marcado

(Créditos fotográficos: Archivo WCS Chile)
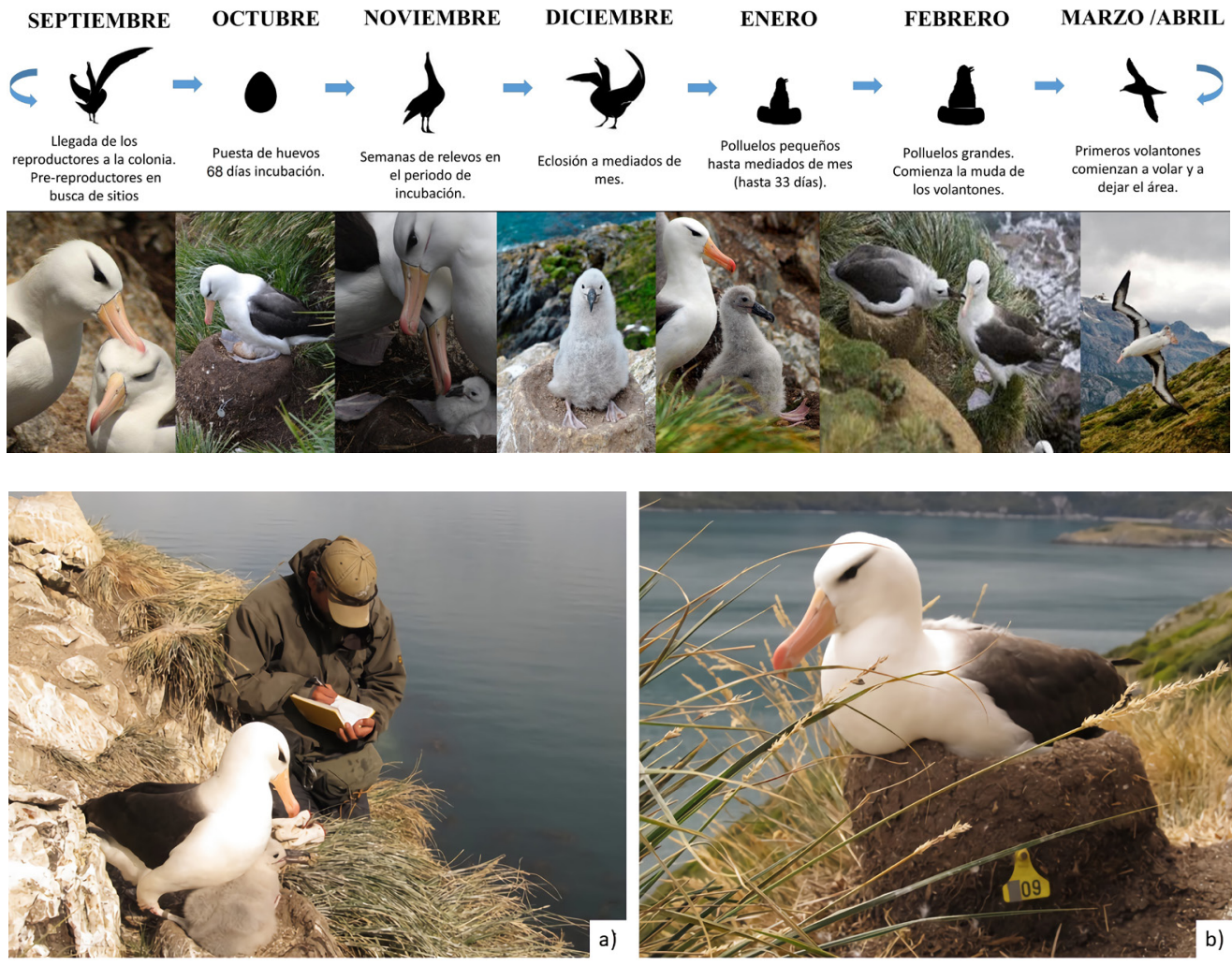

\section{Anillamiento}

El anillamiento se realizó solo entre las temporadas 2009 y 2015, se marcaron individuos con anillos metálicos de $18 \mathrm{~mm}$, facilitados por el Servicio Agrícola y Ganadero (SAG), para determinar la tasa de retorno al islote. Cada temporada se registraron los individuos anillados en temporadas previas.

\section{RESULTADOS}

La temporalidad de ocurrencia de las expediciones realizadas no fue homogénea durante el periodo de estudio, debido que tanto a restricciones climáticas como financieras se realizaron en días y meses distintos en cada temporada (Tabla 1). La colonia fue visitada al menos una vez en el mes de octubre y noviembre, cinco veces durante el mes de diciembre, en dos oportunidades en enero y febrero. Si bien los registros no son directamente comparables, dificultando la estimación del tamaño de la colonia, si nos permiten describir aspectos claves sobre la biología reproductiva de esta colonia.

El número de nidos activos al inicio de la temporada reproductiva osciló entre un máximo de 44 en el año 2010 y cero durante visitas realizadas en 2014, 2015 y 2016 (Tabla 2). El número total de nidos disponibles registrado (nidos vacíos y ocupados) varió entre 25 y 78 (mediana = 48; Tabla 2). Si bien las fechas de las campañas de trabajo no fueron constantes a lo largo de los 
TABLA 1. Expediciones realizadas al Islote Albatros, AMCP-MU Seno Almirantazgo, entre las temporadas 2009 y 2016. El año de la temporada corresponde al año de postura de huevos.

\begin{tabular}{|c|c|c|}
\hline Temporada & Fecha expedición & Fecha monitoreo Islote \\
\hline 2009 & 15 al 19 de diciembre del 2009 & 17 diciembre \\
\hline 2010 & 11 al 19 de diciembre del 2010 & 14 diciembre \\
\hline 2011 & 5 al 15 de enero del 2012 & 7 - 11 enero \\
\hline 2012 & 8 al 15 de diciembre del 2012 & 12 -14 diciembre \\
\hline 2014 & 21-25 febrero del 2015 & 25 febrero \\
\hline \multirow{3}{*}{2015} & 13 de noviembre del 2015 & 13 de noviembre \\
\hline & 20 de diciembre del 2015 & 20 de diciembre \\
\hline & 11 de febrero del 2016 & 11 de febrero del 2016 \\
\hline \multirow{3}{*}{2016} & 23-28 de octubre del 2016 & 23 - 26 de octubre \\
\hline & 11 de diciembre del 2016 & 11 de diciembre \\
\hline & 21 de enero del 2017 & 21 de enero del 2017 \\
\hline
\end{tabular}

TABLA 2. Número de nidos disponibles totales (vacíos y activos), nidos activos e individuos adultos de albatros de ceja negra por temporada y fecha de monitoreo en el Islote Albatros, Seno Almirantazgo.

\begin{tabular}{ccccc}
\hline \multirow{2}{*}{ Temporada } & $\begin{array}{c}\text { Fecha monitoreo } \\
\text { Islote }\end{array}$ & $\begin{array}{c}\text { Nidos } \\
\text { Disponibles totales }\end{array}$ & $\begin{array}{c}\text { Nidos } \\
\text { activos }\end{array}$ & N $^{\circ}$ Adultos \\
\hline 2009 & 17 diciembre & 48 & 29 & 96 \\
\hline 2010 & 14 diciembre & 62 & 44 & 124 \\
\hline 2011 & $7-11$ enero & 33 & 31 & 66 \\
\hline 2012 & $12-14$ diciembre & 25 & 25 & 50 \\
\hline 2014 & 25 febrero & Sin dato & 0 & 120 \\
\hline 2015 & 13 de noviembre & 78 & 24 & 281 \\
\hline 2015 & 20 de diciembre & 78 & 6 & 0 \\
\hline 2015 & 11 de febrero del 2016 & 78 & 0 & 40 \\
\hline 2016 & $23-26$ de octubre & 45 & 20 & 40 \\
\hline 2016 & 11 de diciembre & 45 & 20 & 0 \\
\hline 2016 & 21 de enero del 2017 & 45 & 0 & 24 \\
\hline
\end{tabular}

años, los registros obtenidos sugieren que la abundancia de ejemplares en la colonia, disminuyó entre 2009-2012 y 2015-2016 (Kruskal-Wallis = 3,43; $p=0.0641$ ).

La presencia de huevos se registró en los meses de octubre (2016) y noviembre (2015), observándose un total de 20 y 24 respectivamente. En estos meses no se registró la presencia de polluelos. Durante las expediciones realizadas en los meses de diciembre fue posible encontrar nidos con presencia de huevos o polluelos, siendo la temporada 2010 en la cual se observó el mayor número de huevos (13) y polluelos (31) (Fig. 4). El número inicial de huevos fue estimado considerando el total de nidos registrados para la colonia: mediana $=48$ (rango $=25-78$ ). La presencia de polluelos recién eclosionados a mediados de diciembre de las temporadas 2009, 
Fig. 4 .

Número máximo de huevos y polluelos registrado en la colonia

del islote Albatros

seno Almirantazgo.

Estos registros

fueron realizados

en distintos meses, durante las temporadas reproductivas entre el 2009 al 2016. Según el mes que se visitó la colonia, fue posible encontrar presencia sólo de huevos o de huevos $y$ /o polluelos.

Fig. 5.

Comparación de número de huevos y polluelos durante los monitoreos realizados en el mes de diciembre de 5 temporadas. $\mathrm{N}=$ número total de nidos activos (presencia de huevos y/o polluelos).

Fig. 6.

Tasa de sobrevivencia de huevos en azul y polluelos en rojo para todas las temporadas combinadas, asumiendo que la eclosión ocurre el 14 de diciembre.

El número inicial de huevos corresponde a la mediana [min-max] del total de nidos disponibles en la colonia (ver Resultados).
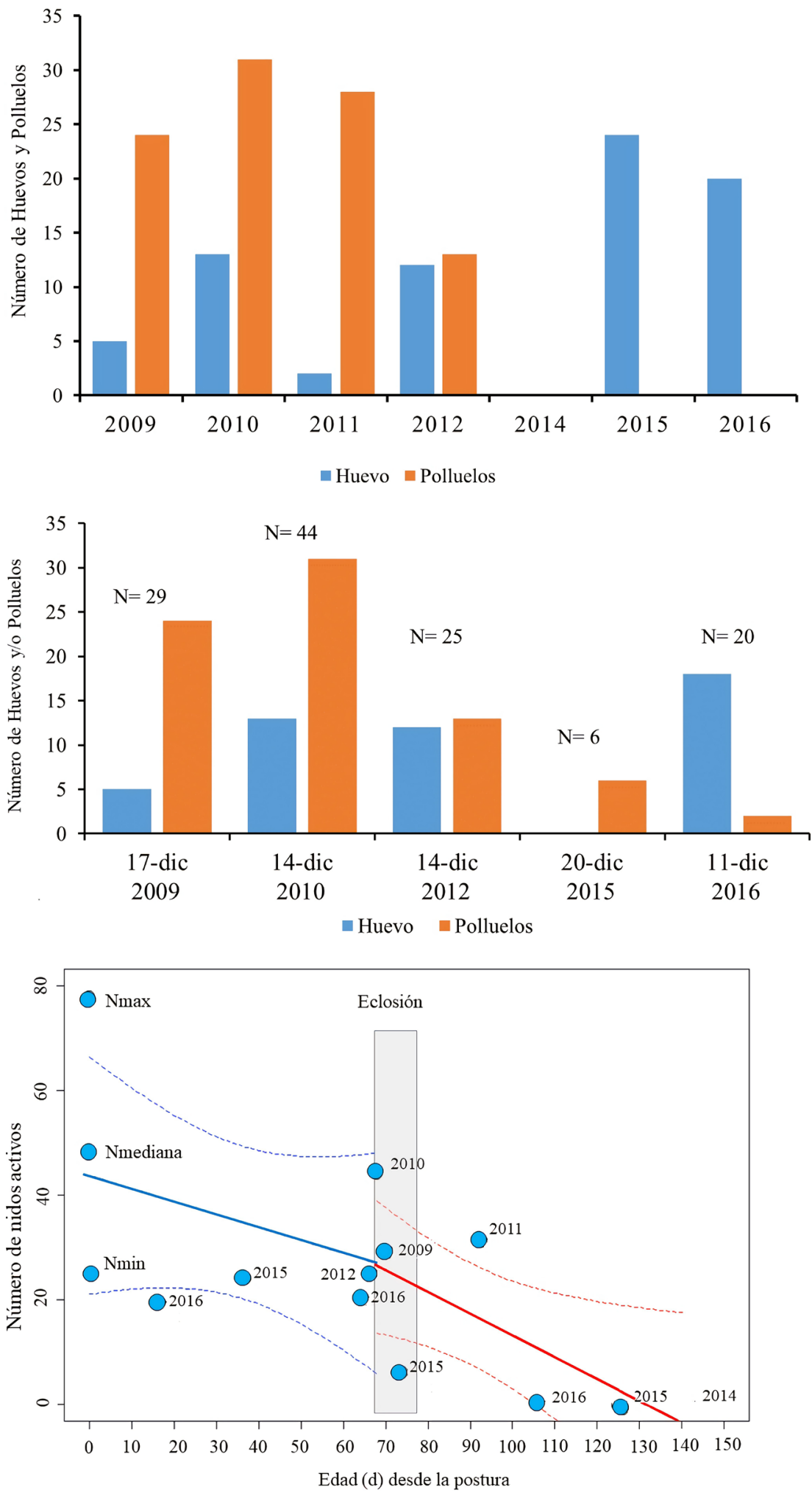
TABLA 3. Porcentaje de recaptura basado en el número de individuos anillados y recapturados por fecha y temporada.

\begin{tabular}{ccccc}
\hline Temporada & Fecha & $\mathbf{N}^{\circ}$ anillados & $\begin{array}{c}\mathbf{N}^{\circ} \text { anillados } \\
\text { recapturados }\end{array}$ & $\begin{array}{c}\text { Porcentaje de } \\
\text { recaptura }\end{array}$ \\
\hline 2009 & 17 diciembre & 48 & 0 & 0 \\
\hline 2010 & 14 diciembre & 70 & 15 & 31 \\
\hline 2011 & 7 enero & 9 & 18 & 30 \\
\hline 2012 & 12 diciembre & 44 & 6 & 4 \\
\hline 2014 & 25 febrero & 17 & 3 & 2 \\
\hline 2015 & 13 noviembre & 0 & 4 & 2 \\
\hline
\end{tabular}

2010, 2012, 2015 y 2016 ( 14-dic, Fig. 5) nos indica que la postura ocurre durante la primera quincena del mes de octubre. La ausencia total de huevos y polluelos el año 2014 coincidió con la visita más tardía realizada a la colonia, con fecha 25 de febrero. Esto fue similar a lo observado durante las visitas realizadas el 11 de febrero de 2016 y el 21 de enero de 2017 (Tabla 2).

La realización de estas 11 expediciones, durante las temporadas 2009 y 2016, permitió evaluar el éxito reproductivo de la colonia. En ambas temporadas se observó una alta mortalidad de polluelos posterior a la fecha media de eclosión estimada para la colonia ( 14 -dic). La tasa de eclosión fue de 62\% (rango: 27-72\%), con un tamaño inicial de 44 (rango: 21-66) huevos (Fig. 6). La tasa de sobrevivencia de los pollos sugiere que la media de individuos eclosionados fue de 26 (rango: 14-39), con una tasa de sobrevivencia de $0 \%$ a los 65 días de edad ( 17 febrero; Fig. 6). Los datos obtenidos, sin embargo, indican que ningún polluelo sobrevivió a los 38 días de edad (hacia el 21 de enero).

\section{Anillamiento}

Se anilló un total de 188 albatros de ceja negra durante siete temporadas (Tabla 3). La tasa de recaptura de los individuos anillados fue variable y estuvo afectada por las fechas de las expediciones (Fig. 7). Durante la temporada 2009/2010 se registró un ejemplar previamente anillado en New Island (islas Falkland/Malvinas). Este individuo fue anillado siendo polluelo en febrero de 2004 por el British Antarctic Survey (P. Catry, comunicación personal, agosto de 2011). Por otro lado, uno de los individuos marcados en el islote Albatros fue observado nidificando en las islas Falkland/Malvinas durante noviembre de 2018 (P. Catry, comunicación personal, noviembre 2018).

\section{DISCUSIÓN}

En este trabajo se presentan nuevos antecedentes sobre el tamaño, fenología y éxito reproductivo de la colonia de albatros de ceja negra del islote Albatros, a partir de un monitoreo más sistemático. Estos antecedentes resultan valiosos para el manejo y la conservación de la especie en el seno Almirantazgo, considerando que se trata de la única colonia de albatros de ceja negra en el mundo que está ubicada dentro de aguas interiores. 
Fig. 7.

Porcentaje de tasa de recaptura de ejemplares anillados de albatros de ceja negra, en función de la fecha de las campañas realizadas cada temporada.

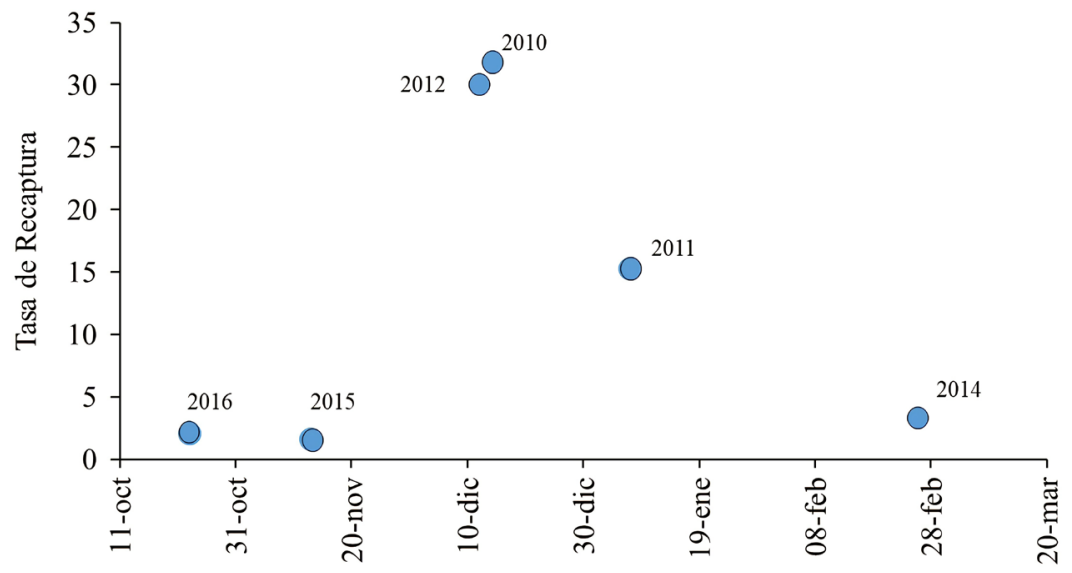

Recaptura de individuos anillados

El tamaño medio de la colonia, basado en los nidos activos, fluctuó considerablemente en función de la fecha de realización de las expediciones. Durante siete temporadas reproductivas fueron detectados, en los meses de octubre y diciembre, entre 20 y 44 nidos activos, estimando la presencia de 44 (rango: 21-66) parejas reproductivas para este período. Dentro del período de estudio, se observó una menor abundancia de nidos activos en las temporadas 2015 y 2016, respecto a 2009-2012. Las primeras estimaciones poblacionales disponibles para esta colonia indicaron la presencia de 50 parejas en enero de 2003 (Aguayo et al. 2003), mientras que en 2006 se observaron 65 parejas (Robertson et al. 2007), valores claramente más altos que los encontrados en nuestro estudio.

En cuanto a la fenología de la colonia, las visitas realizadas en distintos momentos a lo largo del ciclo reproductivo de la especie permitieron determinar la fecha aproximada de eclosión de los huevos en la colonia, que sería alrededor del 14 de diciembre. Basados en este antecedente, y el período de incubación conocido para la especie (68 días), la temporada reproductiva en el islote Albatros comenzaría durante el mes de septiembre, con el regreso y la instalación de los adultos para armar los nidos, para iniciar el período de postura durante la primera quincena del mes de octubre. Esta temporalidad coincide con la descrita para las islas Crozet (Weimerskirch et al. 1986) y las islas Georgias del sur (Prince et al. 1994; Tickell \& Pinder,1975), siendo 5-7 días más tardía que para Diego Ramírez (Arata, J., datos sin publicar).

La tasa de sobrevivencia de huevos y pollos fue estimada utilizando los datos combinados para todas las temporadas (Fig. 6). La tasa de eclosión estimada (media $=62 \%$ ) es similar a la observada en Bird Island (media $=67,5 \%$ ) por Prince y colaboradores (1994). Por el contrario, la sobrevivencia de los polluelos que logran eclosionar parece disminuir drásticamente durante la etapa de guarda y no se logró registrar ningún polluelo vivo después de la primera quincena de enero, en ninguno de los tres períodos más tardíos de muestreo, realizados en las temporadas de estudio (Tabla 2, Fig. 6). Dentro del ciclo reproductivo de la especie, la sobrevivencia de los polluelos, desde la eclosión hasta el final de la etapa de guarda (moda= 23, rango: 11-33 días de edad; (atry et al. 2010), es crítica para el éxito reproductivo de la colonia, siendo prácticamente despreciable durante la postguarda (Catry et al. 2006). Los resultados de este estudio contrastan 
con las tasas de sobrevivencia de polluelos a la edad de 70 días estimadas para New Island (media = 58.8\%), Bird Island (47.5\%) y Diego Ramírez (78.5\%) (Catry et al. 2010; Arata, J., datos sin publicar). En particular, las visitas periódicas realizadas a la colonia durante las temporadas 2015 y 2016 sugieren una alta mortalidad durante los primeros días de vida de los polluelos. Estos antecedentes sugieren que la colonia de albatros de ceja negra del islote Albatros estaría disminuyendo, debido a otros factores que afectan el éxito reproductivo de las parejas que allí anidan.

La reducción en la tasa de recaptura en esta colonia particularmente en los últimos años (Fig. 7) podría reflejar asimismo un potencial abandono de la colonia por parte de adultos. La observación de un ejemplar anillado en las islas Falkland/Malvinas en el islote Albatros y, posteriormente, el registro de un ejemplar anillado del islote Albatros anidando en las islas Falkland/Malvinas, sugiere un intercambio de individuos entre ambas colonias. Asimismo, esto sugiere que la disminución en la viabilidad del islote como área de nidificación podría llevar a una potencial emigración de individuos desde el mismo.

Hasta el momento se desconocen cuáles son las variables que podrían afectar el éxito reproductivo de los ejemplares de la colonia del islote Albatros. El éxito reproductivo del albatros de ceja negra ha sido vinculado a cambios en la disponibilidad de alimento, mortalidad de los adultos debido a captura incidental por pesca, inexperiencia de los padres, y depredación (Catry et al. 2008; Forster \& Phillips, 2009; Pardo et al. 2017; Prince et al. 1994). Asimismo, se desconoce la existencia de mortalidad ocasionada por pesca o por efectos ambientales (e.g. el Niño, la Niña, cambio climático) durante la temporada reproductiva para esta colonia.

Durante la temporada 2014 se registró por primera vez la presencia de un visón (Neovison vison) en el islote Albatros, especie exótica invasora que tiene efectos devastadores en colonias de aves acuáticas (Schüttler et al. 2009) y ha sido reportada en la zona centro y sur de Tierra del Fuego e isla Navarino (Anderson et al. 2006; Crego et al. 2014; Rozzi \& Sherriffs, 2003). La presencia de esta especie podría eventualmente explicar el éxito reproductivo nulo de los albatros de ceja negra del islote Albatros durante las temporadas 2014 a 2016. Asimismo, se ha registrado la presencia de otros predadores, como traros o caranchos (Caracara plancus), tiuques (Milvago chimango) y cóndores (Vultur gryphus), los cuales podrían estar contribuyendo con la falla reproductiva.

Considerando tanto estos antecedentes como las fluctuaciones observadas en nuestro estudio, se pone de manifiesto la necesidad de mantener un esfuerzo de monitoreo sistemático, mejorando su temporalidad, para evaluar la tendencia y la viabilidad de esta colonia. Tal esfuerzo debiera considerar la continuidad de los censos; el anillamiento de adultos reproductores, juveniles y polluelos para desarrollar estudios demográficos; la identificación y mitigación de posibles amenazas, como el visón u otros factores de presión, que puedan afectar la supervivencia y el éxito reproductivo de los ejemplares. En este contexto, es importante considerar la disminución que sufrió el éxito reproductivo durante las últimas temporadas, y se identifica la urgente necesidad de determinar cuáles son las causas que podrían haberla provocado, como así también si están relacionadas o no con la presencia del visón.

Los resultados obtenidos demuestran que la colonia de albatros de ceja negra del islote Albatros es pequeña y, por lo tanto, vulnerable a los factores de presión que puedan estar afectando a esta área. El monitoreo constante de la colonia y la identificación de estos factores 
de presión permitirían diseñar y evaluar, adaptativamente, medidas de manejo y conservación que contribuyan a mitigarlos y favorecer su desarrollo a través del tiempo, contribuyendo así al desarrollo del futuro plan de manejo de la AMCP-MU Seno Almirantazgo, y que serán determinantes para la viabilidad de este objeto de conservación del área protegida.

\section{AGRADECIMIENTOS}

A las embarcaciones de Cruceros Australis, Northanger, Turismo MaryPaz II, M/V Forrest y la Armada de Chile, como así también a los dueños de Caleta María, por su colaboración y apoyo logístico para las actividades de campo. Reconocemos y valoramos a quienes nos apoyaron en terreno, especialmente a Marcela Uhart, Catherine Dougnac, Jorge Acevedo, Richard Cudney y al equipo de guardaparques del Parque Karukinka. Agradecemos la colaboración e intercambio de información sobre los individuos anillados a Paulo Catry del British Antarctic Survey. Finalmente, queremos agradecer a The David \& Lucile Packard Fundation y el Ministerio del Medio Ambiente, quienes generosamente contribuyeron a financiar parte de este estudio.

\section{LITERATURA CITADA}

Aguayo, A., Acevedo, J., \& Acuña, P. (2003). Nuevo sitio de anidamiento del Albatros ceja negra, Diomedeo melanophris Temmink 1828, en el Seno Almirantazgo, Tierra del Fuego, Chile. Anales del Instituto de la Patagonia (Chile), 31, 91-96.

Anderson, C., Rozzi, R., Torres-Mura, J., McGehee, S., Sherriffs, M., Schüttler, E., \& Rosemond, A. (2006). Exotic vertebrate fauna in the remote and pristine sub-Antarctic Cape Horn Archipelago, Chile. Biodiversity and Conservation, 15, 3295-3313.

Arata, J., Robertson, G., Valencia, J., \& Lawton, K. (2003). The Evangelistas islets, Chile: a new breeding site for blackbrowed albatrosses. Polar Biology, 26, 638-647.

Arata, J., Croxall, J., Huin, N., Nicholls, D., Phillips, R., Quintana, F., Robertson, C., Robertson, G., Trathan, P., \& Falabella, V. (2009). Albatros. En V. Falabella, C. Campagna, y J. Croxall (Eds.), Atlas del Mar Patagónico: Especies y espacios (pp. 76-129). Wildlife Conservation Society \& BirdLife International.

Arata, J., Vila, A., Matus, R., Droguett, D., Silva, C., Falabella, V., Robertson, G., \& Haro, D. (2014). Use and exploitation of channel waters by the black-browed albatross. Polar Biology, 37(4), 565-571.

Campioni, L., Granadeiro, J., \& Catry, P. (2017). Albatrosses prospect before choosing a home: intrinsic and extrinsic sources of variability in visit rates. Animal Behaviour, 128, 85-93.

Catry, P., Phillips, R., Forcada, J., \& Croxall, J. (2006). Factors affecting the solution of parental dilemma in albatrosses: at what age should chicks be left unattended? Animal Behaviour 72, 383-391.

Catry, P., Lecoq, M., \& Strange, I. (2008). Population growth and density, diet and breeding success of striated caracaras Phalcoboenus australis on New Island, Falkland islands. Polar Biology, 31, 1167-1174.

Catry, P., Phillips, R., Forster, I., Matias, R., Lecoq, M., Granadeiro, J., \& Strange, I. (2010). Brood-guarding duration in black-browed albatrosses Thalassarche melanophris: temporal, geographical and individual variation. Journal of Avian Biology, 41, 460-469.

Crego, R., Jiménez, J., Soto, C., Barroso, O., \& Rozzi, R. (2014). Tendencias poblacionales del visón norteamericano invasor (Neovison vison) y sus principales presas nativas desde su arribo a isla Navarino, Chile. Boletín de la Red Latinoamericana para el Estudio de Especies Invasoras, 4(1), 4-18.

Croxall, J., Prince, P., Rothery, P., \& Wood, A. (1998). Population changes in albatrosses at South Georgia. In G. Robertson y R. Gales (Eds.), Albatross biology and conservation (pp. 69-83). Surrey Beathy \& Sons Pty Ltd.

Foro para la Conservación del Mar Patagónico y áreas de influencia. (2019). Informe del Primer Taller Regional de Evaluación del Estado de Conservación de Especies para el Mar Patagónico según criterios de la Lista Roja de UICN: Mamíferos Marinos. V. Falabella y C. Campagna (Eds.). 
Forster, I.P \& Phillips, R.A.(2009).Influence of nest location, density and topography on breeding success in the BlackBrowed albatros Thalassarche Melanophris. Marine Ornithology 37: 213-217.

Marín, M., \& Oehler, D. (2007). Una nueva colonia de anidamiento para el albatros de ceja negra (Thalassarche melanophrys) para Chile. Anales del Instituto de la Patagonia (Chile), 35(1), 29-33

Moreno, C., y Robertson, G. (2008). ¿Cuántos albatros de ceja negra, Thalassarche melanophrys (Temminck, 1828) anidan en Chile? Anales del Instituto de la Patagonia (Chile), 36(1), 89-92. http://doi.org/10.4067/S0718$686 \times 2008000100008$

Nevoux, M., Weimerskirch, H., \& Barbraud, C. (2007). Environmental variation and experienced-related differences in the demography of the long-lived black-browed albatross. Journal of Animal Ecology, 76, 159-167. http:// doi.org/10.1111/j.1365-2656.2006.01191.x

Ojeda, J., Suazo, C., \& Rau, J. (2011). Ensamble estacional de aves marinas en la pesquería del palangre artesanal de la merluza austral Merluccius australis, en canales subantárticos de Chile. Revista de Biología Marina y Oceanografía, 46(3), 443-451. http://doi.org/10.4067/S0718-19572011000300013

Pardo, D., Forcada, J., Wood, A.G., Tuck, G.N., Ireland, L., Pradel, R., Croxall, J.P., \& Phillips, R.A. (2017). Additive effects of climate and fisheries drive ongoing declines in multiple albatross species. PNAS, 114 (50), E10829-E10837. http://doi.org/10.1073/pnas.1618819114.

Prince, P., Rothery, P., Croxall, J., \& Wood, A. (1994). Population dynamics of Black-browed and Grey-headed albatrosses Diomedea melanophris and D. chrysostoma at Bird Island, South Georgia. Ibis, 136(1), 50-71. http://doi. org/10.1111/j.1474-919X.1994.tb08131.x

Robertson, G., Moreno, C., Lawton, K., Arata, J., Valencia, J., \& Kirkwood, R. (2007). An estimate of the population size of Black-browed (Thalassarche melanophrys) and Grey-headed (T. chrysostoma) Albatrosses breeding in the Diego Ramírez Archipelago, Chile. The Emu- Austral Ornithology, 107(3), 239-244. http://doi.org/10.1071/MU07028

Robertson, G., Moreno, C., Arata, J., Candy, S., Lawton, K., Valencia, J., Wienecke, B., Kirkwood, R., Taylor, P., \& Suazo, C. (2014). Black-browed albatross numbers in Chile increase in response to reduced mortality in fisheries. Biological Conservation, 169, 319-333.

Robertson, G., Wienecke, B., Suazo, C.G., Lawton, K., Arata, J.A., \& Moreno, C. (2017). Continued increase in the number of black-browed albatrosses (Thalassarche melanophris) at Diego Ramirez, Chile. Polar Biology, 40,_1035-1042.

Rozzi, R., \& Sherriffs, M. (2003). El visón (Mustela vison SHREBER, CARNIVORA, MUSTELIDAE), un nuevo mamífero exótico para la Isla Navarino. Anales del Instituto de la Patagonia, 31, 97-104.

Schüttler, E., Klenke, R., McGehee, S., Rozzi, R., \& Jax, K. (2009). Vulnerability of groundnesting waterbirds to predation by invasive American mink in the Cape Horn Biosphere Reserve, Chile. Biological Conservation, 142, 1450-1460.

Tickell, W., \& Pinder, R. (1975). Breeding biology of the Black-browed Albatross Diomedea melanophris and Greyheaded Albatross D. chrysostoma at Bird Island, South Georgia. Ibis, 117(4), 433-451. http://doi.org/10.1111/j.1474919X.1975.tb04237.x

Vila, A., Pushel, N., Rodríguez, M., Guijón, R., \& Kusch, A. (2017). Propuesta Área Marina Costera Protegida de Múltiples Usos Seno Almirantazgo. Tierra del Fuego, Región de Magallanes y de la Antártica Chilena. Informe Técnico. Wildlife Conservation Society -Chile

Wakefield, E., Phillips, R., Trathan, P., Arata, J., Gales, R., Huin, H., Robertson, G., Waugh, S., Weimerskirch, H., \& Matthiopoulos, J. (2011). Habitat preference, accessibility, and competition limit the global distribution of breeding black-browed Albatrosses. Ecological Monographs, 81(1), 141-167. http://doi.org/10.1890/09-0763.1

Weimerskirch, H., Jouventin, P., \& Stahl, J. (1986). Comparative Ecology of the 6 Albatross Species Breeding on the Crozet Islands. Ibis, 128, 195-213.

Weimerskirch, H., Clobert, J., \& Jouventin, P. (1987). Survival in five southern albatrosses and its relationship with their life history. Journal of Animal Ecology, 65, 1043-1055.

Wolfaardt, A. (2012). An assessment of the population trends and conservation status of Blackbrowed Albatrosses in the Falkland Islands. Joint Nature Conservation Committee (JNCC), July 2012. Available at: http://www. epd.gov.fk/wpcontent/uploads/An\%20assessment\%20of\%20the\%20conservation\%20status\%20of\%20 BBA\%20in\%20the\%20Falkland\%20lslands_July\%202012_Final.pdf. Accessed 24 de septiembre de 2013). 
Apéndice: Afiliación de cada autor

\begin{tabular}{cc}
$\begin{array}{c}\text { Número } \\
\text { afiliación }\end{array}$ & Nombre de la institución y/o organización Afiliación \\
\hline 1 & Wildlife Conservation Society Chile, Balmaceda 586, Punta Arenas, Chile \\
2 & $\begin{array}{l}\text { Programa de Magíster en Ciencias, mención en Manejo y Conservación de Recursos Naturales en } \\
\text { Ambientes Subantárticos. Universidad de Magallanes, Punta Arenas, Chile. }\end{array}$ \\
3 & Far South Expeditions, Punta Arenas, Chile. \\
4 & Centro de Rehabilitación de Aves Leñadura, Punta Arenas, Chile \\
5 & Asociación de Investigadores del Museo de Historia Natural Río Seco, Punta Arenas, Chile. \\
6 & Association of Responsible Krill harvesting companies, ARK. Toronto, ON, Canadá \\
\hline & Autor \\
\hline $\begin{array}{l}\text { Daniela Droguett } \\
\text { Alejandro Vila }\end{array}$ & 1,2 \\
Alejandro Kusch & 1,3 \\
Ricardo Matus & 4 \\
Benjamín Cáceres \\
Javier Arata
\end{tabular}

